\title{
Study of Closed-Loop Model Reference Adaptive Control of Smart MicroGrid with QNU and Recurrent Learning
}

\author{
Vladimír Malý ${ }^{1,5}$, Martin Veselý ${ }^{2,5}$, Peter M. Beneš ${ }^{3,5}$, Petr Neuman ${ }^{4}$, Ivo Bukovský 5,* \\ 'VM ENGINEERING s.r.o. Klášterec nad Ohří, Czech Republic \\ ${ }^{2}$ FMV-DESIGN s.r.o. Trutnov, Czech Republic \\ ${ }^{3}$ Corporate Technology Development Center, SIEMENS s.ro. Praha, Czech Republic \\ ${ }^{4}$ Neureg sdr., Praha, Czech Republic \\ ${ }^{5}$ CTU in Prague, Faculty of Mechanical Engineering, Czech Republic
}

\begin{abstract}
An adaptive quadratic polynomial neural unit (QNU) controller for optimization of a conventional Smart Microgrid control loop is studied and proposed. The parameters associated with the studied grid plants are considered to be known in this study, with the fact that the load is unknown and time-variant. A sample-by-sample real-time recurrent learning algorithm of an additional QNU controller is derived, with its performance tested and discussed as a result of this paper.
\end{abstract}

Keywords: Smart Grids, Microgrids, Diesel Engine Generators (DEG), Photovoltaic panels (PV), Wind Turbine Generators (WTG), Flywheel Energy Storage System (FESS), Battery Energy Storage System (BESS), QNU Controller, Linear and Non-linear Controllers.

\section{Introduction}

Smart Grids (SGs) can be deemed as rather complex, large systems, in which smart control is one of the necessary conditions for SG realization (Neuman, 2011, 2012, 2014). In late 1990's, the main issues related to Distributed Grids (DG), nowadays referred to as MicroGrids, were widely considered by the working groups of the International Council on Large Electric Systems (CIGRE) and the International Conference and Exhibition on Electricity Distribution (CIRED) in their review reports (Chowdhury et al., 2009).

Smart control methods and other tasks in SGs often involve computational intelligence tools such as artificial neural networks (Mori and Awata, 2006) or fuzzy logic (Bevrani et al., 2012). MicroGrids (MGs) are subsystems of complex Smart Grids. Till now, Phasor Measurement Unit (PMU) technology is often categorized as a significant tool to implement Smart Grids (Mitani et al., 2014). With regards to their control techniques, adaptive reference model approaches have proven their relevance as such in the works (Gibson et al., 2013; Narendra and Valavani, 1979). A key contribution of this paper is the introduction of a novel closed-loop model reference adaptive control scheme. For efficient real-time learning algorithms as such that of the gradient descent algorithm (GD) and the Levenberg-Marquardt (L-M) batch training algorithm, higher order neural units (HONUs), have proven to be computationally efficient in achieving adequate convergence in square error whilst achieving desirable control performance for both non-linear unknown systems as well as linear systems of SISO structure. The conception of quadratic neural units (i.e. 
a second order $\mathrm{HONU}$ ) along with their application to both linear as well as non-linear engineering processes have been studied throughout the works (Bukovsky et al., 2010; Gupta et al., 2003 \& 2013). A more recent application of HONUs for realtime adaptive control may be found in the work (Bukovsky et al., 2015) where HONUs were applied in extension to the previously employed conventional control loops for successful optimisation on various SISO engineering processes.
To demonstrate this novel approach for application to SG design, we derive the recurrent adaptation of a closed loop for control of a sub-component of a microgrid model (adopted from (Bevrani et al., 2012)). In this case study, two serial plants representing DEG, MG, FESS, and BESS subsystems are classically shown in a general PID closed loop in Fig. 1, where the individual grid components are generalized as known linear plant transfer functions, with respective parameters detailed in Table 1.

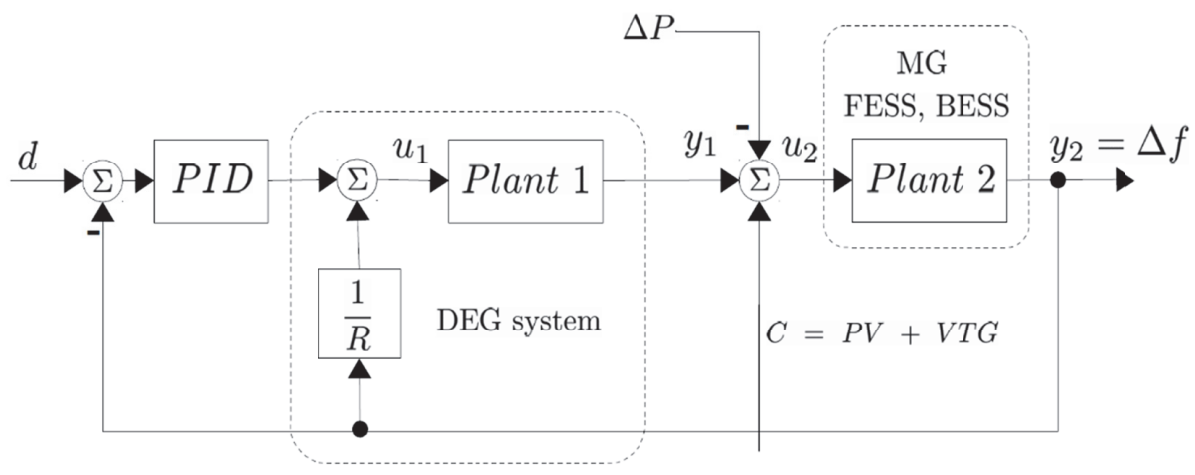

Fig. 1: Closed loop of Smart Micro Grid part as given in Fig.1 with details in Tab. 1 (adopted from (Bevrani et al., 2012)).

Plant 2 (MG, FESS,BESS)
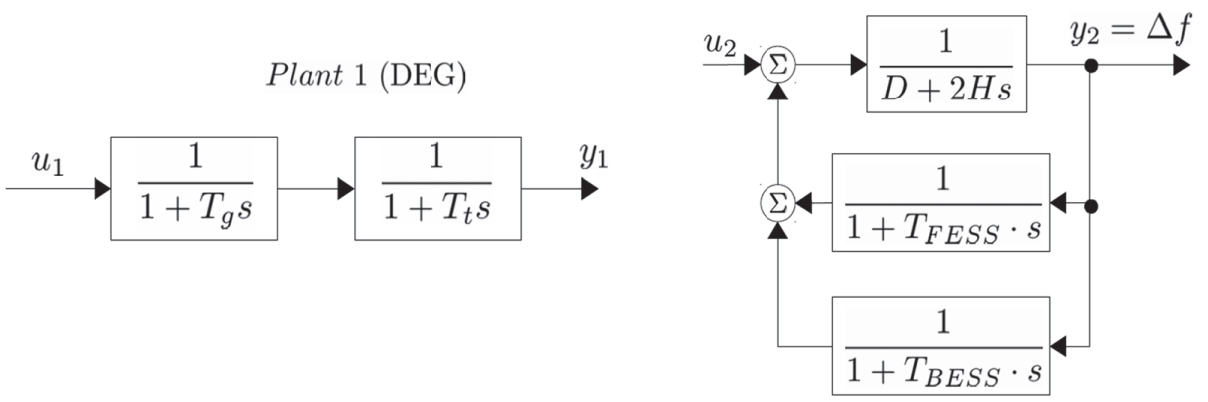

Fig. 2: Models of Smart Grid part as given in Fig. 1 with details in Tab. 1 (adopted from (Bevrani et al., 2012)).

Tab. 1: Sub-part of microgrid model configuration as adopted from (Bevrani et al., 2012).

\begin{tabular}{|c|c|c|c|}
\hline $\begin{array}{l}\text { Plant } 1 \\
\text { DEG system [s] }\end{array}$ & $\begin{array}{l}\text { Plant } 2 \\
\text { MG system }\end{array}$ & FESS[S] & BESS [s] \\
\hline $\begin{array}{c}T_{t}=0.4 \\
T_{g}=0.08 \\
R=3[\mathrm{~Hz} / \mathrm{pu}]\end{array}$ & $\begin{array}{l}D=0.015[\mathrm{pu} / \mathrm{Hz}] \\
2 H=0.1667[\mathrm{pus}]\end{array}$ & $T_{F E S S}=0.1$ & $T_{B E S S}=0.1$ \\
\hline
\end{tabular}

\section{Discrete Time Control Loop with QNU Controller}

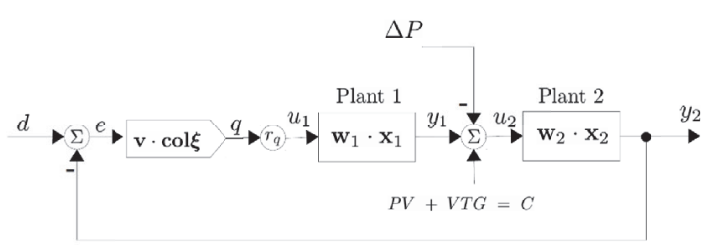

Fig. 3: Control loop with adaptive QNU controller in a discrete-time form, where $d=$ const. and $\Delta P$ is the immeasurable load. 
The discrete time model of the control loop for the purpose of discrete-time controller learning may be designed as follows. The grid frequency offset $\Delta f=y_{2}$ is the output of a serial block of two linear plants as in Fig. 3, which may be given via the following relation.

$$
\Delta f(k)=y_{2}(k)=\mathbf{w}_{2} \cdot \mathbf{x}_{2}(k)
$$

where the input vector is

$$
\mathbf{x}_{2}(k)=\left[\begin{array}{c}
y_{2}(k-1) \\
y_{2}(k-2) \\
\vdots \\
y_{2}\left(k-n_{y 2}\right) \\
u_{2}(k-1) \\
u_{2}(k-2) \\
\vdots \\
u_{2}\left(k-n_{u 2}\right)
\end{array}\right]=\left[\begin{array}{c}
y_{2}\left(k-n_{y 2}: k-1\right) \\
u_{2}\left(k-n_{u 2}: k-1\right)
\end{array}\right]=\left[\begin{array}{c}
\mathbf{y}_{2} \\
\mathbf{u}_{2}
\end{array}\right]
$$

and where $\mathbf{w}_{2}$ is a long row vector of $n_{y 2}+n_{u 2}=n_{2}$ adaptive parameters (neural weights) based on a priori approximate of the discrete time plant parameters. Further, the control input $u_{2}$ may be calculated via the following summation.

$$
u_{2}(k)=y_{1}(k)+C(k)+\Delta P(k)
$$

where $\Delta P$ is the immeasurable grid load and $C$ stands for other external grid input components (that are beyond the scope and hence, not considered in depth within this paper).

Similarly, the output of the serial two-plant block before the load input is calculated may be given as follows

$$
y_{1}(k)=\mathbf{w}_{1} \cdot \mathbf{x}_{1}(k),
$$

where the input vector is

$$
\mathbf{x}_{1}(k)=\left[\begin{array}{l}
y_{1}\left(k-n_{y 1}: k-1\right) \\
u_{1}\left(k-n_{u 1}: k-1\right)
\end{array}\right]=\left[\begin{array}{l}
\mathbf{y}_{1} \\
\mathbf{u}_{1}
\end{array}\right],
$$

and where $\mathbf{w}_{2}$ is a long vector of $n_{y 2}+n_{u 2}=n_{2}$, based on the a priori approximate plant parameters. Furthermore, the control error $e$, may be defined as follows

$e=d-y_{2}$,

where the set point $d=d(t)$, is a constant.
Instead of a conventional PID controller, we propose an adaptively tuned quadratic neural unit (QNU), i.e., second-order non-linear polynomial controller of the following structure

$q(k)=\mathbf{v} \cdot \operatorname{col} \xi(k)$

where the long-column vector $\operatorname{col} \xi$ of polynomial input terms in the sense of a QNU may be defined as vector

$\operatorname{col} \xi=\left[\left\{\xi_{i} \cdot \xi_{j}\right\}\right] ; i=0 \ldots n_{y 2}+1, j=i \ldots n_{y 2}+1$

and where the $\xi_{i}, \xi_{j}$ are elements of the controller input vector $\boldsymbol{\xi}$ that is defined as follows

$$
\xi=\left[\begin{array}{c}
\xi_{0} \\
\xi_{1} \\
\xi_{2} \\
\vdots \\
\xi_{n_{y 2}+1}
\end{array}\right]=\left[\begin{array}{c}
1 \\
q(k-1) \\
y_{2}(k) \\
y_{2}(k-1) \\
\vdots \\
y_{2}\left(k-n_{y 2}+1\right)
\end{array}\right],
$$

and $\mathbf{v}$ in equation (7) is the long-row vector of adaptable parameters with respect to the adaptive controller defined as follows

$\mathbf{v}=\left[\left\{v_{i, j}\right\}\right] ; i=0 \ldots n_{\xi}, j=i \ldots n_{\xi}$,

Where $n_{\xi}=2+n_{y 2}$. Finally, the output of the QNU controller (7), according to Fig. 3 multiplied with inclusion of an additional gain, may be given as follows

$u_{1}(k)=r_{q} \cdot q(k)$

where $r_{q}$ is an additional (optional) static gain that may be incorporated for example, in cases where a QNU may result in large output magnitudes corresponding to low static gains of the controlled system.

\section{Reference Model Design for The Closed Loop Scheme}

For the adaptive closed loop scheme in Fig. 3, we propose the discrete time reference model via Z-transfer function as follows

$Y_{r e f}(z)=\frac{r_{o} \cdot\left(z^{2}-z\right)}{\left(r_{o} \cdot T_{d}-1\right) \cdot z+1} \Delta P(z)$, 
where its parameters $T_{d}$ and $r_{o}$ define the decay rate and gain respectively. To demonstrate the effectiveness in terms of suppression to an introduced disturbance. Fig. 4 illustrates the step responses of the designed reference model to an introduced disturbance under various configurations of decay rate and gain.

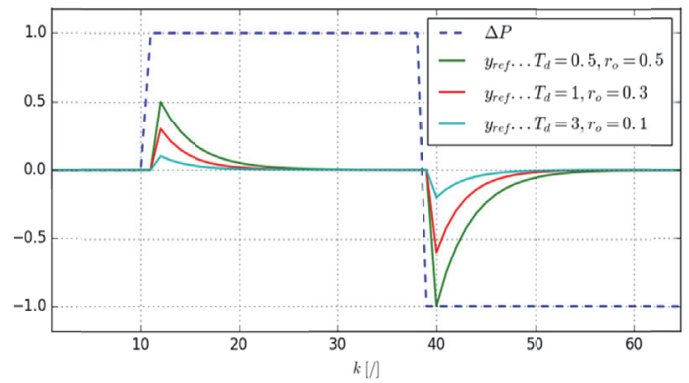

Fig. 4: Step responses of the closed loop reference model (12) to a disturbance $\Delta P$ under various configurations.

\section{Controller Learning Rule}

The parameters of the QNU controller are proposed to be adapted by the gradient descent learning rule as

$\mathbf{v}(k+1)=\mathbf{v}(k)+\Delta \mathbf{v}$,

where we adaptively enforce the control loop (Fig. 3) to adopt the behavior of the reference model (12) as follows

$\Delta \mathbf{v}=-\frac{\mu}{2} \frac{\partial e_{r e f}^{2}(k)}{\partial \mathbf{v}}=\mu \cdot e_{r e f}(k) \frac{\partial y_{2}(k)}{\partial \mathbf{v}}$,

where the reference error is defined as $e_{\text {ref }}=y_{\text {ref }}-y_{2}$, and where

$\frac{\partial y_{2}(k)}{\partial \mathbf{v}}=\mathbf{w}_{2} \cdot \frac{\partial \mathbf{x}_{2}(k)}{\partial \mathbf{v}}$

where the partial derivatives of control input $\mathbf{u}_{2}$ in $\mathbf{x}_{2}$ are according to (3) and Fig. 3 as follows

$\frac{\partial u_{2}}{\partial \mathbf{v}}=\frac{\partial\left(y_{1}+C+\Delta P\right)}{\partial \mathbf{v}}=\frac{\partial y_{1}}{\partial \mathbf{v}}$

because neither $C$ nor $\Delta P$ depends on the QNU controller weights $\mathbf{v}$. In (16) we can see that the unknown load, when assumed as the additive perturbation (3), does not affect the learning rule of the QNU controller.

Further derivations from (16) follow as

$\frac{\partial y_{1}}{\partial \mathbf{v}}=\mathbf{w}_{1} \cdot \frac{\partial u_{1}}{\partial \mathbf{v}}=\mathbf{w}_{1} \cdot r_{q} \cdot \frac{\partial q}{\partial \mathbf{v}}$,

where according to (7)

$$
\frac{\partial q}{\mathbf{v}}=\operatorname{col} \xi^{T}+\mathbf{v} \cdot \frac{\partial \operatorname{col} \xi}{\partial \mathbf{v}},
$$

where the quadratic term derivatives may be calculated as follows

$$
\frac{\partial \operatorname{col} \xi}{\partial \mathbf{v}}=\frac{\partial \xi_{i}}{\partial \mathbf{v}} \cdot \xi_{j}+\xi_{i} \cdot \frac{\partial \xi_{j}}{\partial \mathbf{v}}
$$

Finally the partial derivatives of vector $\boldsymbol{\xi}$ may then be given as follows

$$
\frac{\partial \xi(k-\imath)}{\partial \mathbf{v}}=\left[\begin{array}{c}
\mathbf{0} \\
\frac{\partial \mathbf{q}(k-\imath-1)}{\partial \mathbf{v}} \\
\frac{\partial \mathbf{y}_{2}(k-\imath)}{\partial \mathbf{v}} \\
\partial \mathbf{y}_{2}(k-\imath-1) \\
\vdots \\
\frac{\partial \mathbf{y}_{2}\left(k-\imath-n_{y 2}+1\right)}{\partial \mathbf{v}}
\end{array}\right]
$$

that recurrently develop from zero initial conditions via (15) - (20).

\section{Closed-Loop Performance of the Proposed Adaptive Control}

This section illustrates the resulting adaptive controller tuning in a closed loop and compares the controller performance of a linear adaptive controller in Fig. 5 and of quadratic non-linear controller (QNU) in Fig. 6. From the two figures, it can be concluded that the algorithm results in an adequate convergence of both architectures towards the desired reference model set point values. With the quadratic neural unit architecture in particular, exhibiting smoother adhesion to the desired reference model under introduced perturbations in comparison to the LNU controller output. 

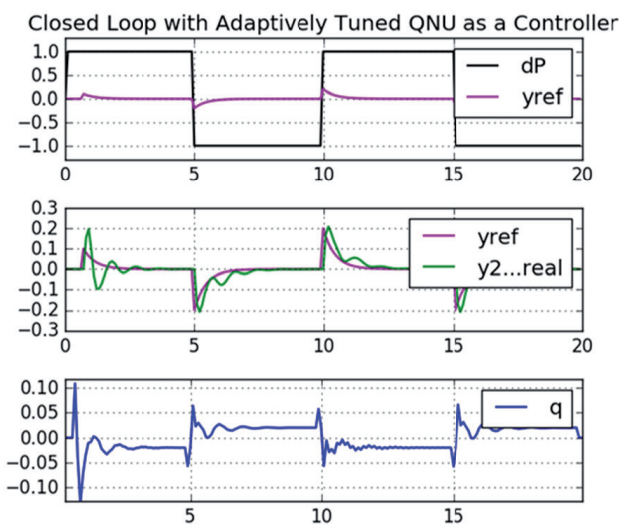

Fig. 5: Step response of a closed control loop to disturbance steps $\triangle P$ during last adaptation epoch of (non-linear) QNU controller.
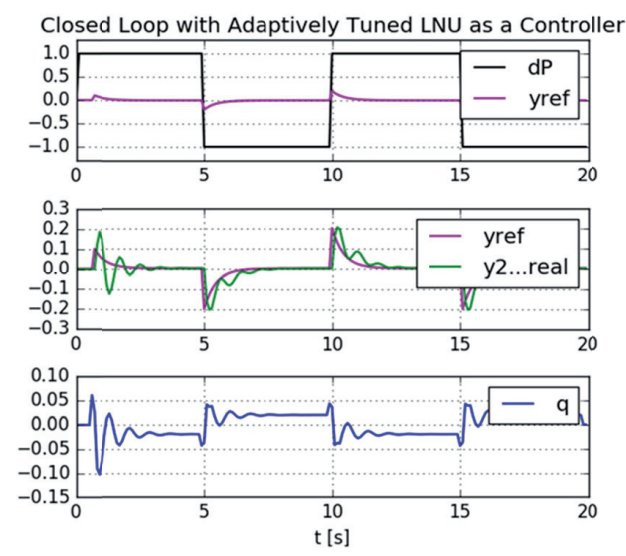

Fig. 6: Step response of a closed control loop to disturbance steps $\triangle P$ during last adaptation epoch of LNU controller.

\section{Conclusions}

Throughout this paper, a novel approach of model reference adaptive control via a quadratic polynomial neural unit (QNU) controller for control of Power Systems in a closed loop, with immeasurable load and constant set point was presented. The potentials of which is of utmost importance and relevance towards Smart Microgrid control applications. The presented simulation results have shown that learning (adaptation) extends in a closed loop and also converged for a non-linear controller architecture. These results demonstrate the correctness of the chosen development direction and authorizes the necessity for further research within this field. The resulting sampling of the whole discrete closed control loop of the $50 \mathrm{~Hz}$ phasor model is $0.1 \mathrm{sec}$, which correlates to the speed of whole system response, further justifying the applicability of the method. An aim for further research, is directed towards a comparison of linear versus non-linear controllers from the viewpoint of control quality for different controlled systems and operating conditions.

\section{Acknowledgments}

V. Malý, M. Veselý and P. M. Beneš were supported by the Grant Agency of the (zech Technical University in Prague, grant No. SGS15/189/0HK2/3T/12. I. Bukovský acknowledges support from the EU Operational Programme Research, Development and Education, and from the Center of Advanced Aerospace Technology (CZ.02.1.01/0.0/0.0/16_019/0000826), Faculty of Mechanical Engineering, (zech Technical University in Prague.

\section{References and Notes}

[1] Bevrani, H., Habibi, F., Babahajyani, P., Watanabe, M., Mitani, Y., 2012. Intelligent Frequency Control in an AC Microgrid: Online PSO-Based Fuzzy Tuning Approach. IEEE Trans. Smart Grid 3, 1935-1944. doi:10.1109/TSG.2012.2196806

[2] Bukovsky, I., Benes, P., Slama, M., 2015. Laboratory Systems Control with Adaptively Tuned Higher Order Neural Units, in: Silhavy, R., Senkerik, R., Oplatkova, Z.K., Prokopova, Z., Silhavy, P. (Eds.), Intelligent Systems in Cybernetics and Automation Theory, Advances in Intelligent Systems and Computing. Springer International Publishing, pp. 275-284.

[3] Bukovsky, I., Homma, N., Smetana, L., Rodriguez, R., Mironovova, M., Vrana, S., 2010. Quadratic neural unit is a good compromise between linear models and neural networks for industrial applications, in: 2010 9th IEEE International Conference on Cognitive Informatics (ICCI). Presented at the 2010 9th IEEE International Conference on Cognitive Informatics (ICCI), pp. 556-560. doi:10.1109/ COGINF.2010.5599677

[4] Chowdhury, S., Chowdhury, S.P., Crossley, P., 2009. Microgrids and active distribution networks, IET renewable energy series. Institution of Engineering and Technology, Stevenage.

[5] Gibson, T.E., Annaswamy, A.M., Lavretsky, E., 2013. Adaptive Systems with Closed-loop Reference Models: Composite control and observer feedback. IFAC Proc. Vol. 46, 440-445. doi:10.3182/20130703-3-FR-4038.00144

[6] Gupta, M.M., Jin, L., Homma, N., 2003. Static and dynamic neural networks: from fundamentals to advanced theory. Wiley, New York.

[7] Madan M. Gupta, Ivo Bukovsky, Noriyasu Homma, Ashu M. G. Solo, Zeng-Guang Hou, 2013. Fundamentals of Higher Order Neural Networks for Modeling and Simulation, in: Ming Zhang (Ed.), Artificial Higher Order Neural Networks for Modeling and Simulation. IGI Global, Hershey, PA, USA, 
pp. 103-133.

[8] Mitani, Y., Kudo, T., Satake, A., Basri, K.H., 2014. Monitoring the Wide Area Power System Dynamics by Phasor Measurement Units Based on Campus WAMS Strategy. IFAC Proc. Vol. 47, 2273-2278. doi:10.3182/20140824-6-ZA-1003.00771

[9] Mori, H., Awata, A., 2006. A Hybrid Method of Clipping and Artificial Neural Network for Electricity Price Zone Forecasting. IEEE, pp. 1-6. doi:10.1109/PMAPS.2006.360234

[10] Narendra, K.S., Valavani, L.S., 1979. Direct and indirect model reference adaptive control. Automatica 15, 653-664. doi:10.1016/0005-1098(79)90033-5

[11] Neuman, P., Pokorny, M., Weiglhofer, W., 2014. Principles of Smart Grids on the generation electrical and thermal energy and control of heat consumption within the District Heating Networks. Proc. of 19th IFAC World Congress 2014, Cape Town, South Africa.

[12] Neuman, P., 2012. Smart Grids applications for improved Power System Control. Proc. of IFAC Symposium 2012, Toulouse, France.

[13] Neuman, P., 2011. Smart Grids methods of Transmission System Operators for improved Power System Control. Proc. of 18th IFAC World Congress 2011, Milan, Italy.

\section{Biographical notes}

Vladimír Malý, Ing.: born in 1979, graduated from (zech Technical University in Prague, Bc. specialization Information and Automation Technology (year of graduation: 2010); Ing. specialization Power Engineering (year of graduation: 2014). He is founder of VM ENGINEERING, s.r.o. and current PhD. student in Technical Cybernetics at Czech Technical University in Prague, Czech Republic. 